\title{
Crossing a Multi-Lane Street: Irrelevant Cars Increase Unsafe Behavior
}

\author{
Baurès Robin $^{* \dagger} \quad$ Daniel Oberfeld ${ }^{\dagger}$ Heiko Hecht ${ }^{\dagger} \quad$ Viola Cavallo* \\ (*)Ifsttar, Labroratoire de Psychologie de la Conduite, France \\ $(†)$ Department of Psychology, Johannes Gutenberg-Universität Mainz, Mainz, Germany \\ E-mail: robin.baures@ifsttar.fr,oberfeld@uni-mainz.de,hecht@uni-mainz.de, \\ viola.cavallo@ifsttar.fr
}

\begin{abstract}
Before crossing a road or an intersection, road users have to determine among the surrounding traffic whether or not they have enough time to safely complete their maneuver. Temporal judgments have been investigated for single cars approaching an intersection, however, close to nothing is known about how street-crossing decisions are being made when several vehicles are simultaneously approaching in two adjacent lanes. We conducted a simulator experiment in which observers indicated whether or not they had enough time to complete safe street crossing. Traffic gaps were presented either with a single or two oncoming cars on different lanes, in such a way that in all cases, only the shortest gap was taskrelevant. Nevertheless, street-crossing decisions were found to be also influenced by the task-irrelevant longer gap, observers being more willing to cross the street when having to judge two gaps simultaneously compared to only one gap. Consequences of this unsafe behavior are discussed.
\end{abstract}

\section{Introduction}

Road crossing is a dangerous situation in which an accurate perception of the time-to-arrival (TA, that is the time remaining before the approaching car reaches the intended crossing path) of the surrounding vehicles is crucial. It has usually been shown that age of the observer [1] and vehicle's velocity or distance [2] are important factors in the TA perception. However, little attention has been paid on the number of lanes to pass through, and therefore the number of gaps that have to be judged concurrently. A gap is the temporal interval between two cars, which may or may not be sufficient for the pedestrian or driver to accomplish his maneuver. This lack of experimental data is cumbersome because city centers abound with streets in which road users (pedestrians, cyclists and drivers) may have to cross multiple lanes, and hence to accept or refuse more than one gap in the same time. The TA perception and consequent crossing decision in a multiple-lanes street may significantly differ from those obtained in a one-lane street.

Indeed, previous studies showed that the human visual system faces the greatest difficulties to determine several TAs in the same time. This has been shown both for relative TA estimation (assessing which of $\mathrm{N}$ object will arrive first) and for absolute TA estimation (assessing the exact time at which several objects are taken to arrive). In the former case, TA judgments were affected by set-size, with a decrease in accuracy as set-size increased [3], whereas in the latter case, TA estimations have been shown to interfere one with the other, in an asymmetric fashion $[4,5]$ : while the TA of the first-arriving object is correctly estimated compared to a one-object condition, the TA of the second-arriving object is significantly overestimated.

Crossing a multiple-lane street is a particular case however. In theory, when having to decide whether the two TAs allow the observer to initiate his maneuver, only the shorter TA should influence the judgment: if the shorter TA is above the observer's crossing time (more a safety margin), then the gap should be accepted. On the contrary, if the TA is below the observer's crossing time (more a safety margin), then the gap should be refused. Note that in both cases, the longer gap is not expected to play any role in the decision. Hence, the crossing decision should be independent of the number of gaps to accept or refuse.

From this observation, three potential outcomes may be predicted.

(1) If the observer is able to make two separate and accurate TA estimates, then the street-crossing decision should not depend of the number of approaching cars. In this situation, for a given TA, street-crossing decisions should not differ between the single-gap and the dual-gap conditions.

(2) Confronted to a more complex situation, the TA estimations may interfere with one another. Consequently, it might results from this interference that the shortest TA would be underestimated. Thus, observers think they

This is an Open Access article distributed under the terms of the Creative Commons Attribution-Noncommercial License 3.0, which permits unrestricted use, distribution, and reproduction in any noncommercial medium, provided the original work is properly cited. 
have less time to cross the street than is actually available.

(3) On the contrary, the interference between the TAs may lead the shorter TA to be overestimated. That is, observers think they have more time to cross the street than is actually available.

Note that while the two first potential outcomes would not affect the observer's safety, compared to the situation with only a single approaching vehicle, the third one implies a very hazardous behavior, which may be an important risk factor when crossing a twolane street.

To summarize, on the basis of the existing literature on concurrent TA perception, three distinct patterns of results can be predicted. We designed an experiment to decide between these hypotheses. We carried out a gap-acceptance experiment in which participants had to indicate whether or not they would drive a car through a two-lane intersection, on which one (singlegap condition) or two (two-gap condition) cars were approaching in adjacent lanes and from the same direction toward the observers.

\section{Material and methods}

\subsection{Subjects}

Fourteen participants ( 5 women, 9 men, age 31.64 years \pm 5.56 (mean \pm SD), min age 25 , max age 43 ) participated voluntarily after giving informed consent according to the procedures approved by the Ethics Committee of the Institut Français des Sciences et Techniques des Transports, de l'Aménagement et des Réseaux.

\subsection{Apparatus and experimental procedure}

The study was conducted using a high-fidelity, realtime driving simulator. Participants were seated in the middle of five $2.50 \times 1.80 \mathrm{~m}$ (height $\times$ length) screens forming an incomplete octagon. Five video projectors were used to back-project the virtual environment onto the screens, at a spatial resolution of $1400 \times 1050$ pixels, and a frame rate of $60 \mathrm{~Hz}$. The device also included a 3D sound-rendition system.

The visual scene was generated using an in-house software library developed at Ifsttar, and consisted of two perpendicular roads crossing in front of the observer, who was waiting at a stop sign.

From the left side of the road, one (single-gap condition) or two (dual-gap condition) groups of vehicles approached toward the intersection (Fig. 1). Each group of vehicles consisted in a first car (gapopening vehicle), and a second car (gap-closing vehicle). Each which could be moving at a constant velocity of $30 \mathrm{~km} / \mathrm{h}$ or $60 \mathrm{~km} / \mathrm{h}$. The first car was placed to reach the intersection after $3 \mathrm{~s}$ of movement. The viewing time before the gap(s) began was therefore constant, and of $3 \mathrm{~s}$. At the end of this viewing time, all the vehicles disappeared from the screen, with the gap-closing vehicle(s) placed to present various TAs.

In the single-gap condition, the temporal value of the gap could be $2,3.5,5,6.5$ or $8 \mathrm{~s}$. In addition, the group of vehicles could be moving in the first or second lane. Ten repetitions were made for each combination, leading participants to perform 200 trials $(2$ lanes $\times 2$ velocities $\times 5$ temporal gap values $\times 10$ repetitions) randomly presented in this single-gap condition. Participants had to indicate whether they would cross the street through the gap, only if they felt they could achieve this maneuver safely.

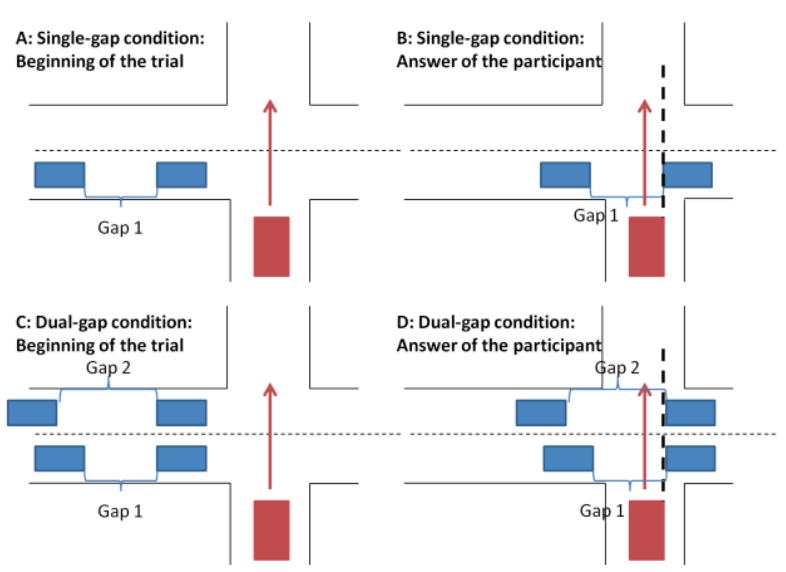

Figure 1: Schematic representation of the task, from a bird's eye point of view, in the single-gap (panels A and $\mathrm{B}$ ) or dual-gap (panels $\mathrm{C}$ and $\mathrm{D}$ ) conditions, at the beginning of the trial (panels $\mathrm{A}$ and $\mathrm{C}$ ) or at the instant when the cars disappeared from the screen and the observers had to give their answer (panels B and D). Blue rectangles represent the approaching cars, and red rectangles the position of the observer. The red arrows represent the desired movement of the observer to cross the street, and the dashed black lines indicate the moment of the disappearance of the vehicles from the screen.

At the end of this first condition, we determined for each participant and in each combination of lane and velocity ( 2 lanes $\times 2$ velocities) the individual temporal gaps for which the participant decided to cross the street in half of the trials (MAG, mean accepted gap). 
In the dual-gap condition, participants faced two groups of vehicles, one in each lane, approaching toward the intersection from the left to the right. Each gap could be moving at a constant velocity of $30 \mathrm{~km} / \mathrm{h}$ or $60 \mathrm{~km} / \mathrm{h}$. The temporal value of one gap was set at the value of the MAG (this gap could be moving either in the first or second lane), while the temporal value of the second gap was a modification of the MAG, termed $\Delta \mathrm{Gap}$, that could be $-50 \%,-25 \%,+25 \%$, or $+50 \%$. Ten repetitions were made for each single combination of lane position, reference gap velocity, second gap velocity and $\Delta \mathrm{Gap}$, leading participants to perform 320 trials ( 2 lanes $\times 2$ reference gap velocities $\times 2$ second gap velocities $\times 4 \Delta$ Gap $\times 10$ repetitions) randomly presented in this dual-gap condition.

\subsection{Data analysis}

Does the presence of two cars rather than only one car systematically influence the decision of the participants to either cross or not cross the road? More specifically, imagine a gap duration for which in the situation with only one car approaching the crossroad the participant is indifferent concerning crossing, that is, pcross $=.5$. If now an additional car is presented on the other lane, with a longer gap duration so that in theory it would be completely irrelevant to the decision, will this second car nevertheless alter the proportion of trials in which the participant decides to cross the road?

To answer this question, we used a Generalized Linear Mixed Model (GLMM) to analyze the effect of the second car on pcross. We fitted a logistic model using a logit link function. In the model, the log odds for crossing were assumed to depend on the shorter gap $\left(\right.$ gap $\left._{\min }\right)$ presented on a given trial, and on the number of cars (one or two) with a subject-specific, randomeffects model approach.

A similar analysis was conducted to determine if the difference between the two gaps influence the crossing decision. Only the data from the two-gap condition entered this analysis. For each participant, the differences between the longer and the shorter gap were classified into three terciles (3-quantiles groups), and subjected to a logistic model using a logit link function. In the model, the log odds for crossing were assumed to depend on the shorter gap ( gap $_{\text {min }}$ ) presented on a given trial, and on the (binned) difference between the two gaps (gap $_{\text {diff; with the }}$ levels short, medium or large).

\section{Results}

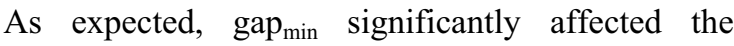
probability that participants accepted to cross the street, $F(1,86.09)=114.37, p<.001$, with a higher probability of a accepting the gap as a function of the increase in gap $_{\text {min }}$ size (see Fig. 2). The number of gaps also significantly influenced the crossing decision, $F(1$, $246)=14.37, p<.001$. As shown in Fig. 2, participants accepted to cross the street for a given gap $_{\min }$ more often in the dual-gap condition rather than in the single-gap condition. For example, for $\operatorname{gap}_{\min }=5 \mathrm{~s}$, participants crossed the street in $40 \%$ of the trials in the single-gap condition, but in $65 \%$ of the trials in the dual-gap condition. Finally, gap min $_{\text {and }}$ ar number did not interact, $F(1,34.79)=0.72, p=.403$. Thus, the slope of the psychometric function relating gap min $_{\text {in }}$ and $\mathrm{p}$ (cross) did not differ between the single-gap and the dual-gap condition.

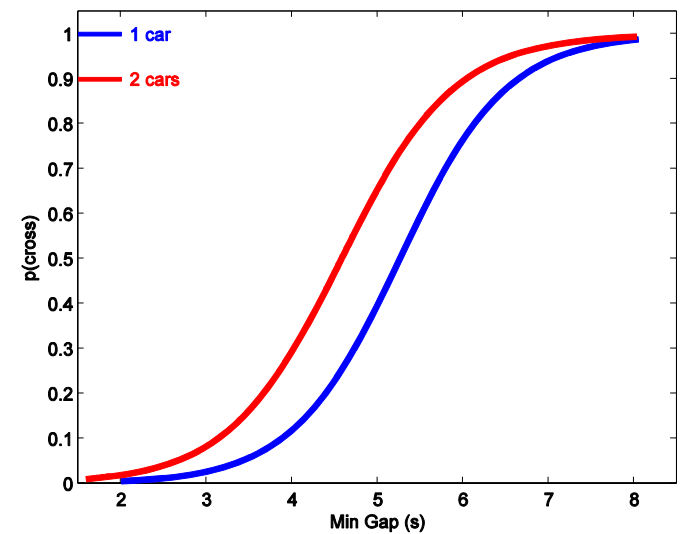

Figure 2: Observers' probability to cross the street as a function of the minimal gap and number of cars (single-gap condition in blue, and dual-gap condition in red). The lines represent the mean crossing probability (aggregated across the different participants) estimated with the GLMM.

Does the sole presence of a second gap lead to such an increase in the rate of street-crossing decisions, or do specific features of the longer gap modulate the effect? For example, the TA estimation of the shortest gap may be biased by a constant offset due to the mere presence of the second gap, or alternatively the bias in the TA estimation may be a function of specific characteristics of the longest gap.

The second GLMM analysis showed that gap min $_{\text {in }}$ significantly influenced the probability that participants decided to cross the street, $F(1,19.55)=181.96, p<$ .001 , with the probability of crossing increasing as a function of the size of gap min $_{\text {. In addition, the analysis }}$ also showed that gap diff $_{\text {had }}$ a significant effect on the 
street-crossing probability, $F(2,148.1)=35.32, p<$ .001. As visible in Fig. 3, for a given value of the shorter gap, participants decided to cross the street more often for longer TAs of the second-arriving car. For example, for gap $_{\min }=5 \mathrm{~s}$, participants crossed the street in $50 \%$ of the trials when the TA of the secondarriving car was only slightly higher than gap min $_{\text {(small }}$ gap $_{\text {diff }}$ ), but in $63 \%$ of the trials when gap diff $_{\text {was }}$ medium, and finally in $75 \%$ of the trials when gap $_{\text {diff }}$

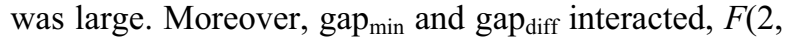
$64.53)=19.29, p<.001$, showing a higher slope for small values of gap $_{\text {diff }}$ than for the medium and large values of gap $_{\text {diff. }}$.

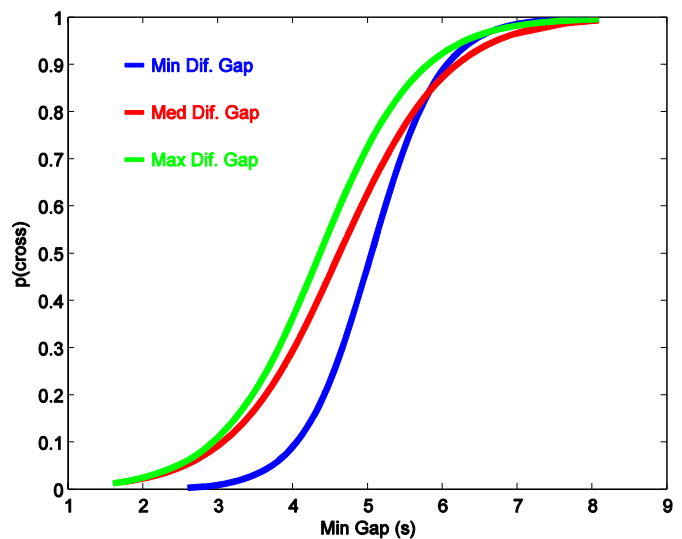

Figure 3: Dual-gap condition. Observers' probability to cross the street as a function of the minimal gap and the difference between the longer and the shorter gap presented on a given trial (minimal difference in blue, medium difference in red, and maximal difference in green). The lines represent the mean crossing probability (aggregated across the different participants) estimated with the GLMM.

\section{Conclusions}

The goal of the present experiment was to determine how street-crossing decisions were influenced by the number of gaps to accept or refuse in the same time. In theory, the number of cars should be irrelevant in such a decision, as only the shorter TA defines the possibility to cross or not the street.

It appears in fact that the street-crossing decision is indeed influenced by the number of gaps to evaluate in the same time. The results indicate that participants are generally more willing to cross the street in the dualgap condition compared to the single-gap condition. In addition, more than the sole presence of a second gap, it was shown that the value of the longer gap plays a role in the decision. Specifically, the influence of the second-arriving car increases as a function of the difference between the two gaps. Hence, for a given value of the task-relevant shorter gap, the taskirrelevant longer gap will increase the probability that the participant decides to cross the street, and this effect is stronger if the second-arriving car will arrive late. This reveals that nonetheless the two TA estimations are not independent, but also that streetcrossing decisions are made based on a weighted average between the two TAs $[6,7]$.

These results challenge our representation of road users' safety behavior. While one would intuitively expect added cars to reduce, or at least keep constant, the positive decisions to cross the street, opposite results are in fact the case. This appears to be a quite counterintuitive and unsafe behavior, and reveals that the relevant TA is overestimated when two cars are approaching the observer. When the TA of an approaching car is being overestimated, observers have less time than thought to carry out the action, and therefore may decide to cross the street despite the lack of time to complete the action. As a consequence, driver and pedestrian safety education might point out the hazard of multiple approaching vehicles.

\section{References}

[1] R. Lobjois, and V. Cavallo. The effects of aging on street-crossing behavior: From estimation to actual crossing, Accident Analysis and Prevention, 41, 259-267 2009

[2] R. Lobjois, and V. Cavallo. Age-related differences in street-crossing decisions: The effects of vehicle speed and time constraints on gap selection in an estimation task, Accident Analysis and Prevention, 39, 934-943, 2007

[3] P. R. DeLucia, and J. B. Novak. Judgments of relative time-to-contact of more than two approaching objects: Toward a method. Perception and Psychophysics, 59, 913-928, 1997

[4] R. Baurès, D. Oberfeld, and H. Hecht. Judging the contact-times of multiple objects: Evidence for asymmetric interference, Acta Psychologica, 134, 363371,2010

[5] R. Baurès, D. Oberfeld, and H. Hecht. Temporal-range estimation of multiple objects: Evidence for an early bottleneck, Acta Psychologica, 137, 76-82, 2011

[6] S. K. Rushton, and J. P. Wann. Weighted combination of size and disparity: a computational model for timing a ball catch, Nature Neuroscience, 2, 186-190, 1999

[7] D. Oberfeld, and H. Hecht. Effects of a moving distractor object on time-to-contact judgments, Journal of Experimental Psychology: Human Perception and Performance, 34, 605-623, 2008 